\title{
Association equilibria for proteins interacted with crowders of short-range attraction in crowded environment
}

\author{
Jiachen Wei* and Fan Song ${ }^{\dagger}$ \\ State Key Laboratory of Nonlinear Mechanics (LNM), \\ Institute of Mechanics, Chinese Academy of Sciences, \\ Beijing 100190, P. R. China \\ School of Engineering Science, \\ University of Chinese Academy of Sciences, \\ Beijing 100049, P. R. China \\ *weijiachen@lnm.imech.ac.cn \\ ${ }^{\dagger}$ songf@lnm.imech.ac.cn
}

Received 12 May 2016

Revised 15 August 2016

Accepted 15 September 2016

Published 5 October 2016

\begin{abstract}
Based on a very simple coarse-grained colloidal model, here we implement an effective hard-sphere theory and numerical simulation to capture the general features of the association equilibria for globular proteins in crowded environment. We measure the activity coefficient, i.e., the deviation from ideal behavior of protein solution, and the crowding factor, i.e., the contribution of crowders to the association equilibria, for proteins in macromolecular crowding. The results show that the association balance in macromolecular crowding depends sensitively on the magnitude of protein-crowder attraction and the relative size of reactant to crowding agent. Since our coarse-grained model is irrelevant to the microscopic details of the molecules, it can be applied to the control of the association equilibria of many globular proteins such as bovine serum albumin, crystallin and lysozyme.
\end{abstract}

Keywords: Globular protein; association equilibria; short-range attraction; macromolecular crowding.

PACS numbers: 61.20.Gy, 82.60.Lf, 87.14.Ee

\section{Introduction}

In living cells, proteins are always found in crowded environment so that their intermolecular interactions strongly depend on the surrounding medium. Abnormal intermolecular interactions can destabilize the association balance, i.e., the equilibrium populations of unbound protein monomers and dimers, resulting in the

†Corresponding author. 
formation of protein aggregates and even phase transition of the bulk system such as phase separation. Many polymerization diseases, such as cataract, sickle cell and Alzheimer's disease, $\stackrel{1-3}{-3}$ are caused by the association of a great number of proteins with abnormal intermolecular interactions. In addition, although the association equilibria of proteins and other types of globular macromolecules in suspension have been found to be significantly affected by intermolecular attraction, $\stackrel{4-6}{-}$ a detailed quantitative description for such process is still absent. Therefore, research on the attraction dependence of the association equilibria for proteins is of great interest.

As one of the simplest coarse-grained approaches to study equation of states of colloidal particles, the scaled particle theory $(\mathrm{SPT})^{7}$ captures the very general features of protein interactions. As a result, it has previously been used to study polymerization diseases of proteins. ${ }^{-\underline{3}} \underline{-3}$ The original SPT only applies to proteins without intermolecular attraction by modeling them as hard-convex particles. ${ }^{1,7}$ To introduce intermolecular interactions, Minton and co-worker ${ }^{4,8-10}$ proposed the effective hard-sphere theory (EHT). According to the assumption of EHT, the effective size of molecules changes as a function of intermolecular interaction (Fig. 2). However, the valid regime for such theoretical approximation has not yet been determined.

Due to its advantages in accurate prediction of the free energy and equilibrated configurations, Monte Carlo (MC) simulation $\underline{11}-15$ has recently been used to obtain the changes in free energy of the binding of two proteins as reactant into a dimer as product in crowded environment. Specifically, Kim and Yethiraj ${ }^{11}$ combined MC simulation with Widom insertion method to test the validity of SPT. In their study, they found that crowder-crowder interaction can barely influence the association equilibria of proteins. However, how the association balance depends on the proteincrowder attraction as well as their size ratio was not investigated.

In this work, we implement MC simulation as well as EHT to investigate the effects of strength and range of intermolecular attractions on association equilibria of globular proteins. We find that EHT is effective when intermolecular attraction is weak or protein concentration is low. The strength of protein-crowder attraction can severely influence the thermodynamic stability of the system, especially at higher protein density. The crowding factor strongly depends on the relative size of reactant protein to crowders, which suggests the possibility of an avalanche of protein associations with the increase of protein sizes. The paper is organized as follows: in Sec. 2 we introduce the model and relevant theories. Section 3 provides results and discussions of the attraction strength, size and range effects on the crowding factor of proteins in macromolecular crowding. Concluding remarks are presented in Sec. 4.

\section{Methods}

\subsection{Model}

Globular proteins are considered as impenetrable hard-spheres devoid of internal degrees of freedom with diameter $D$. According to Noro-Frenkel extended law of 


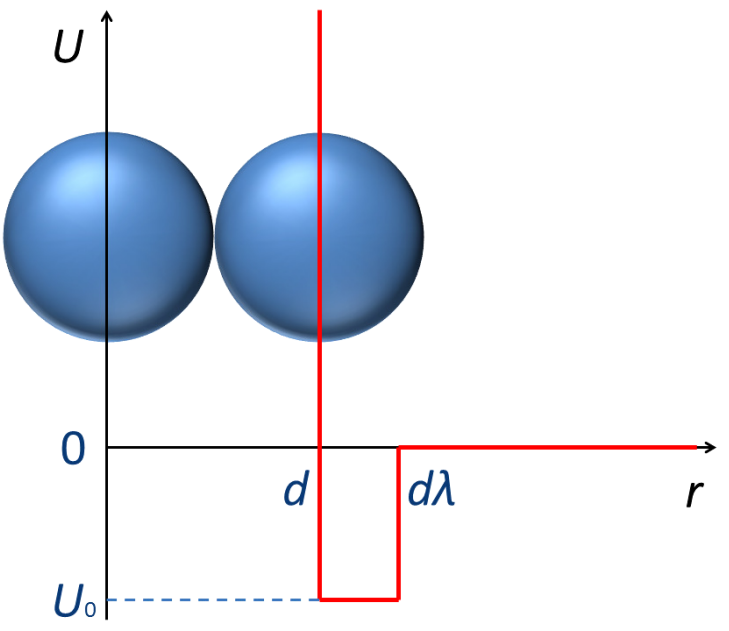

Fig. 1. (Color online) Sketch of SRSW potential.

corresponding states, $\frac{16}{6}$ phase properties are insensitive to the specific shape of the potential for short-range attractive systems. Thus, we set the interaction between two spheres to pairwise short-range square-well (SRSW) potential,,$\frac{16}{23}$ which has already been widely applied to the study of the phase behavior ${ }^{19,23}$ as well as colligative properties ${ }^{6,9,10}$ of protein solutions, and serves as the basic state for understanding the role of attractions in protein association:

$$
U(r)= \begin{cases}\infty & \text { for } r<d, \\ -U_{0} & \text { for } d<r<d \lambda, \\ 0 & \text { for } r>d \lambda,\end{cases}
$$

where $U_{0}$ is the well depth, $\lambda$ controls the well width, $r$ is the particle distance and $d$ represents the contacting distance, see Fig. 1. For single-component system, $d=D$. In our study, $U_{0}$ and $D$ are the units of energy and length, respectively.

For our model, the second virial coefficient, $B_{2}$, is given as

$$
B_{2}=4 \pi \int_{0}^{\infty}\left[1-e^{\frac{-U(r)}{k T}}\right] r^{2} d r
$$

where $T$ is temperature and $k$ is the Boltzmann's constant. Combining Eqs. (1) and $(2)$, we obtain the reduced second virial coefficient, $B_{2}^{*}$, via:

$$
B_{2}^{*}=\frac{B_{2}}{B_{\mathrm{HS}}}=1-\left(e^{\frac{U_{0}}{k T}}-1\right)\left(\lambda^{3}-1\right),
$$

where $B_{\mathrm{HS}}=4 \pi D^{3} / 3$ is the second virial coefficient of hard-sphere system. According to Noro-Frenkel extended law of corresponding states, $\frac{16}{a}$ all short-range attractive systems are characterized by the same thermodynamic properties at the same $B_{2}^{*}$ and $\phi$. 


\subsection{Crowding factor}

Consider two monomers as reactants that polymerize into a dimer as product - all embedded in a crowded environment. The crowding factor (or nonideality factor), $\Gamma$, which measures the contribution of crowders to the association equilibria of the two reactants, is defined as

$$
\Gamma=\frac{K}{K_{0}}
$$

where $K_{0}$ and $K$ denote the equilibrium association constants in dilute and crowded environments, respectively, and are related to the activity coefficients $\gamma_{r}$ and $\gamma_{p}$ for reactant and product:

$$
\ln K-\ln K_{0}=2 \ln \gamma_{r}-\ln \gamma_{p} .
$$

Combining Eqs. (4) and (5), we obtain:

$$
\Gamma=\frac{\gamma_{r}^{2}}{\gamma_{p}}
$$

The activity coefficient, $\gamma$ (for either reactant or product), is associated to the work of inserting another reactant/product particle into the sea of crowders. For system consisting of hard-spheres, according to SPT, we have

$$
\ln \gamma=-\ln (1-\phi)+A_{1} \frac{\phi}{1-\phi}+A_{2}\left(\frac{\phi}{1-\phi}\right)^{2}+A_{3}\left(\frac{\phi}{1-\phi}\right)^{3},
$$

where $\phi$ is the packing fraction of the system, and

$$
\begin{aligned}
& A_{1}=\varsigma^{3}+3 \varsigma^{2}+3 \varsigma, \\
& A_{2}=3 \varsigma^{3}+4.5 \varsigma^{2}, \\
& A_{3}=3 \varsigma^{3},
\end{aligned}
$$

where $\varsigma$ is the ratio of the diameter of the inserted particle to that of background crowders. We assume the product is spherical and the total volume is conserved. Therefore, if reactants and crowders are of the same size, we have $\varsigma_{r}=1$ for reactants and $\varsigma_{p}=\sqrt[3]{2}$ for products. The effect of size polydispersity (i.e., the variation of $\varsigma_{r}$ ) on crowding factor will also be discussed.

\subsection{Effective hard-sphere theory}

Since the SPT only gives $\gamma$ for hard-spheres, we implement EHT to introduce attractive interaction between globular proteins. According to the approximation of $\mathrm{EHT},{ }^{8-10}$ if the interaction between particles is pairwise additive and isotropic, ${ }^{24}$ we have

$$
\phi^{\prime}=\frac{c B_{2}}{8 M},
$$




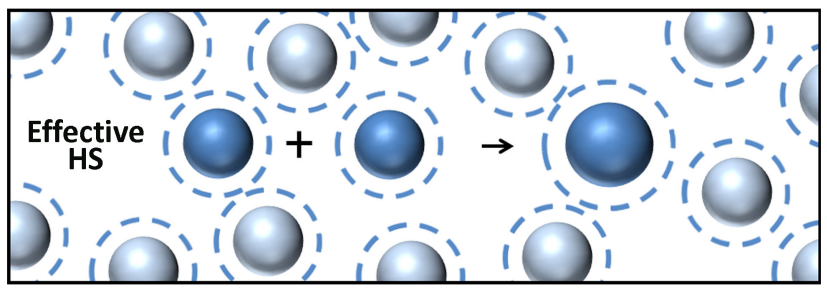

Fig. 2. (Color online) Sketch of two proteins as reactants polymerizing into a dimer as product based on EHT, where dashed lines and blue spheres, respectively, represent the actual and effective sizes of proteins.

where $\phi^{\prime}$ is the effective packing fraction, $c$ is the particle concentration and $M$ is the molar mass of proteins. Therefore, $\phi^{\prime}$ changes as a function of interaction between particles. The larger the attraction between proteins, the smaller the value of $\phi^{\prime}$ (see Fig. 2). By substituting $\phi^{\prime}$ for $\phi$ in Eq. (7), we obtain the activity coefficient of proteins with attraction.

\subsection{Widom insertion method}

We can also numerically obtain the crowding factor to test the effective regime of EHT. Let $p_{r}$ and $p_{p}$ be the probabilities of inserting a reactant and product particle, respectively, into the seas of crowders in conventional Metropolis scheme, we have ${ }^{11}$

$$
\ln \Gamma=\ln p_{p}-2 \ln p_{r} .
$$

While MC simulation is used to produce equilibrated configurations that are nearly independent in NVT ensemble, Widom insertion method ${ }^{11}$ is implemented to obtain $p_{r}$ and $p_{p}$. The initial equilibrated configuration is generated by performing standard Metropolis scheme of 6912 particles of square-well interaction as crowders in a box with periodic boundary condition. Note that we adjust the displacement of the trial move of the particle at every $10^{4} \mathrm{MC}$ simulation steps so that approximately $50 \%$ of the moves will be accepted. Next, for every $10^{5} \mathrm{MC}$ trial moves, we make $10^{7}$ attempts to insert a "ghost" particle (either for reactant or product) at randomly chosen positions. The insertion probabilities are then obtained by averaging over 10 independent runs, each of which consists a total of $10^{8}$ trial moves.

\section{Results and Discussion}

\subsection{Crowding factor}

First, we investigate how crowding factor, $\Gamma$, depends on the packing fraction of crystallins, $\phi$, at different reduced second virial coefficient, $B_{2}^{*}$. It should be noted that the accuracies of both our simulation and theoretical results decrease with the increase of density, therefore we focus more on dilute and moderate concentrations, i.e., $\phi \leq 0.4$, which are common for proteins in physiological environment. We 


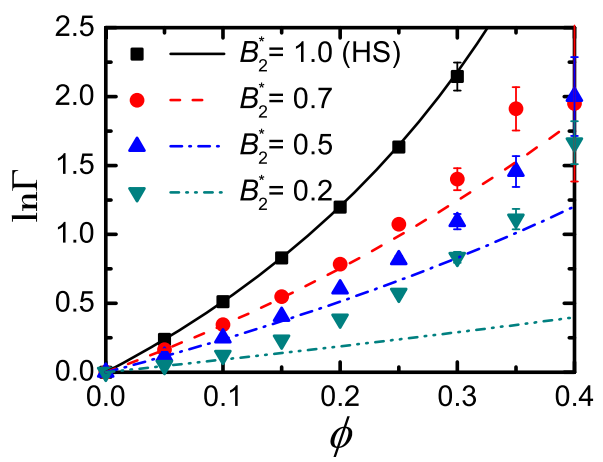

Fig. 3. (Color online) The crowding factor, $\Gamma$, as a function of packing fraction, $\phi$, for SRSW system at different $B_{2}^{*}$ with $\lambda=1.01$ and the diameter ratio of reactants to crowders $\varsigma_{r}=1$. All symbols are numerical results of $\ln \Gamma$ obtained by Widom insertion, while all corresponding lines are derived from EHT.

do not consider any dramatic changes of phase behavior of the bulk, such as phase separation or crystallization, which can hardly take place for thermodynamic states we mainly focus on (i.e., $\phi<0.4$ and $B_{2}^{*}>0.0$ ). Figure 3 shows that, when the reactant and crowders are identical in size, i.e., $\varsigma_{r}=1$, for both numerical and theoretical calculations, $\ln \Gamma$ increases monotonically with the increase of $\phi$ at fixed $B_{2}^{*}$. Since nonspecific attractive interactions always favor reactions that maximize surface exposure, i.e., dissociation of proteins, we always obtain smaller $\ln \Gamma$ for smaller $B_{2}^{*}$ at constant $\phi$. The $\ln \Gamma$ derived from EHT agrees well with that obtained by Widom insertion for purely repulsive system $\left(B_{2}^{*}=1.0\right)$. However, with decrease of $B_{2}^{*}$, results of EHT start to deviate from those of Widom method, especially at higher $\phi$. Nevertheless, although EHT might underestimate the depletion effect at higher density for proteins of strong short-range attraction, it is still quite effective for $\phi<0.1$. We also find that the larger the intermolecular attraction, the more deviation of $\ln \Gamma$ obtained by EHT to that obtained by Widom insertion.

Next, we analyze how $\ln \Gamma$ depends on the ratio of the diameter of inserted reactants to that of background crowders, $\varsigma_{r}$. Figure 4 provides the $\ln \Gamma-\phi$ relation, for SRSW system with $\lambda=1.01$ and $B_{2}^{*}=0.7$, at different values of $\varsigma_{r}$. We can see that, at constant $\phi, \ln \Gamma$ increases with the increase of $\varsigma_{r}$. In addition, since there exists no strong intermolecular attraction $\left(B_{2}^{*}=0.7\right)$, we find $\ln \Gamma$ derived from EHT agrees well with that obtained by Widom insertion at different $\varsigma_{r}$ in both cases.

Figure 5 provides the $\ln \Gamma-\varsigma_{r}$ relation at $\phi=0.1$ for SRSW system with $\lambda=1.01$. We can see that, at fixed $B_{2}^{*}, \ln \Gamma$ increases monotonically with the increase of size ratio. In this case, since the crowding effect is very weak $(\phi=0.1), \ln \Gamma$ derived from EHT agrees well with that obtained by MC simulation, even for system with strong intermolecular attraction $\left(B_{2}^{*}=0.2\right)$. Figure 5 also shows that the growth rate of $\ln \Gamma$ with the increase of reactant diameter is larger for weaker intermolecular attraction. This is due to the cancellation effect of the steric entropic and the attractive enthalpic contributions. 


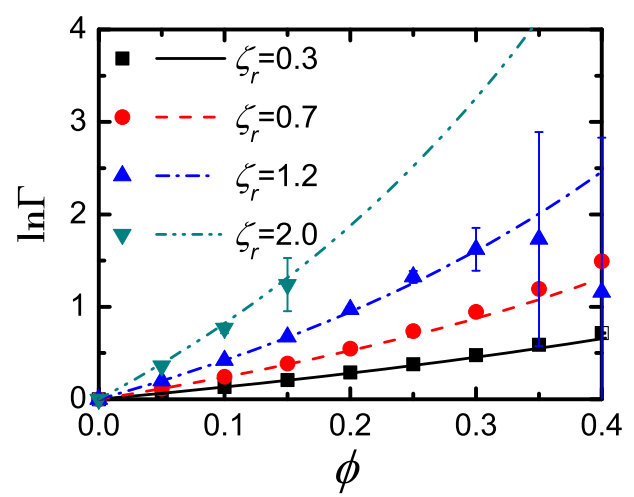

Fig. 4. (Color online) The crowding factor, $\Gamma$, as a function of packing fraction, $\phi$, for SRSW system with $\lambda=1.01$ and $B_{2}^{*}=0.7$, at different diameter ratios $\varsigma_{r}$. All symbols are numerical results of $\ln \Gamma$ obtained by Widom insertion, while all corresponding lines are derived from EHT.

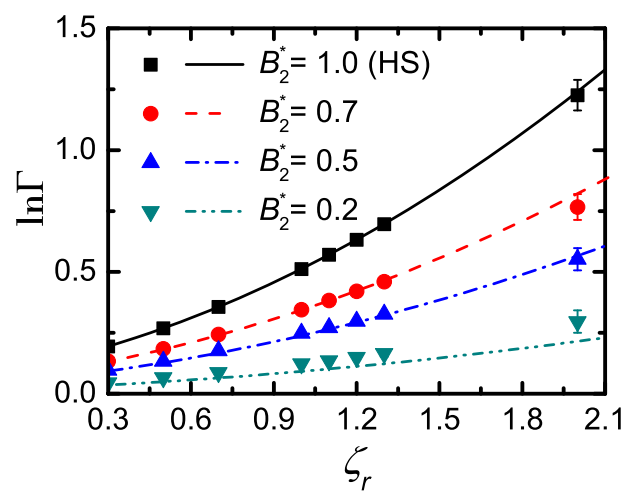

Fig. 5. (Color online) The crowding factor, $\Gamma$, as a function of diameter ratio $\varsigma_{r}$, at fixed packing fraction $\phi=0.1$, for SRSW system at different $B_{2}^{*}$ with $\lambda=1.01$. All symbols are numerical results of $\ln \Gamma$ obtained by Widom insertion, while all corresponding lines are derived from EHT.

In fact, the so-called entropy-enthalpy compensation ${ }^{14}$ denotes the condition at which the steric repulsion and chemical attraction between proteins are canceled out and effectively the crowded environment has no impact on the association equilibria of the proteins (i.e., $\ln \Gamma=0$ ). To determine the critical point at which $\ln \Gamma=0$ is achieved, here we present the crossover behavior of crowding factor, i.e., the $\ln \Gamma-B_{2}^{*}$ relation, in Fig. 6 . We can see that, with the estimation of EHT, $\ln \Gamma=0$ is obtained as long as $B_{2}^{*}=0.0$, see Fig. 6(a). It also shows that $\ln \Gamma$ increases linearly with $B_{2}^{*}$ at fixed packing fraction, which is consistent with former studies on association equilibria of certain types of proteins. ${ }^{12,25}$ However, for $\ln \Gamma$ obtained by Widom insertion, as shown in Fig. 6(b), we find only at $\phi=0.05, \ln \Gamma=0.0$ is achieved when $B_{2}^{*}=0.0$. For higher values of $\phi$, the $\ln \Gamma-\phi$ relation is no longer linear. In addition, although the critical second virial coefficient, $B_{2}^{\text {cri }}$, at which $\ln \Gamma=0.0$ is obtained, decreases with the increase of $\phi$. This indicates that the association 


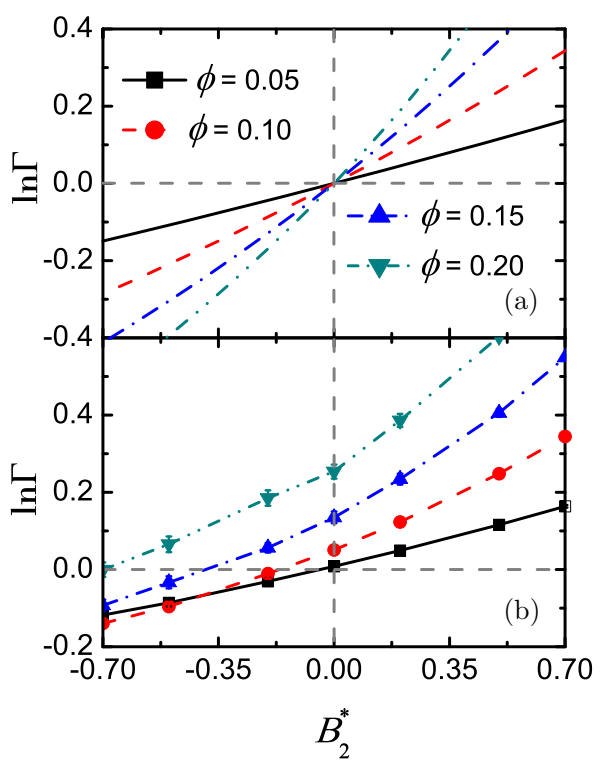

Fig. 6. (Color online) The crowding factor, $\Gamma$, as a function of $B_{2}^{*}$ at different packing fractions $\phi$, for SRSW system with $\lambda=1.01$ obtained by (a) EHT and (b) Widom insertion. We fix the diameter ratio $\varsigma_{r}=1$. All lines in (b) are to guide the eyes. The horizontal dashed line denotes $\ln \Gamma=0.0$. The vertical dashed line gives $B_{2}^{*}=0.0$.

equilibrium of the system is sensitive to the concentration of crowders (or proteins if crowders and reactants are identical).

\subsection{Range effect}

According to Noro-Frenkel extended law of corresponding states, $\frac{16}{}$ the thermodynamic behaviors for short-range attractive system are insensitive to the specific shape of the interaction potential. To test the validity of the law in terms of crowding factor, Fig. 7 presents how $\ln \Gamma$ changes as a function of $\phi$ for SRSW system with $\lambda=1.01$ and $\lambda=1.05$ at different $B_{2}^{*}$. We can see that, at same $B_{2}^{*}$, we obtain very close $\ln \Gamma-\phi$ relation for $\lambda=1.01$ and $\lambda=1.05$, especially at small $\phi$, which confirms the extended law of corresponding states. Moreover, when the attractive interaction is small $\left(B_{2}^{*}=0.7\right.$ and $\left.B_{2}^{*}=0.0\right), \ln \Gamma$ increases monotonically with the increase of $\phi$. When $B_{2}^{*}=-0.7, \ln \Gamma$ first decreases and becomes negative with the increase of $\phi$, which means that system favors the disassociation of proteins at $\phi \sim 0.1$. If protein density is further increased, due to the presence of strong depletion effect, $\ln \Gamma$ starts to increase and becomes positive in value.

Figure 8 shows how $\ln \Gamma$ changes as a function of $\varsigma_{r}$ for SRSW system with $\lambda=1.01$ and $\lambda=1.05$ at different $B_{2}^{*}$. We find $\ln \Gamma$ increases monotonically with the increase of $\varsigma_{r}$ at the same $B_{2}^{*}$. And, the larger the value of $B_{2}^{*}$, the greater is the growth rate of $\ln \Gamma$. Again, we obtain very close $\ln \Gamma-\varsigma_{r}$ relation for $\lambda=1.01$ and $\lambda=1.05$ at same $B_{2}^{*}$, especially when $\varsigma_{r}$ is small in value. 


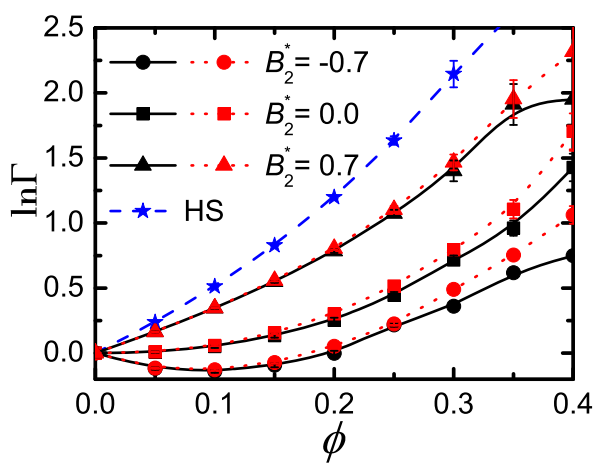

Fig. 7. (Color online) The crowding factor, $\Gamma$, as a function of packing fraction, $\phi$, for SRSW system with $\lambda=1.01$ (black solid lines) and $\lambda=1.05$ (red dotted lines), at different $B_{2}^{*}$. The diameter ratio of reactants to crowders is $\varsigma_{r}=1$. Blue dashed line indicates $\ln \Gamma$ for HS system. All symbols are numerical results of $\ln \Gamma$ obtained by Widom insertion, and the lines are spline fitting curves to guide the eyes.

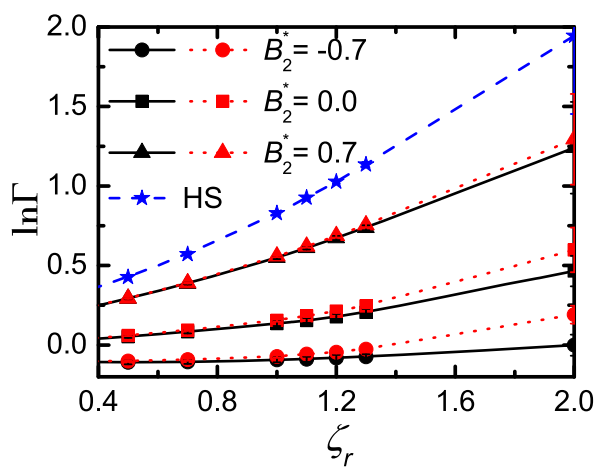

Fig. 8. (Color online) The crowding factor, $\Gamma$, as a function of diameter ratio, $\varsigma_{r}$, at fixed packing fraction $\phi=0.15$, for SRSW system with $\lambda=1.01$ (black solid lines) and $\lambda=1.05$ (red dotted lines), at different $B_{2}^{*}$. Blue dashed line indicates $\ln \Gamma$ for HS system. All symbols are numerical results of $\ln \Gamma$ obtained by Widom insertion, and the lines are spline fitting curves to guide the eyes.

\section{Conclusion}

In this work, we analyzed the effect of protein-crowder attraction on their association equilibria in crowded environment. By comparing MC simulation with theoretical calculation, we find EHT is effective in dilute environment. In addition, EHT can also be applied to systems of moderate or higher density as long as interparticle attraction is weak enough. At fixed packing fraction, we find the crowding factor depends on the second virial coefficient of the system but not the attractive range of the interaction, which confirms the extended law of corresponding states. Both EHT and numerical estimation show that the crowding factor becomes larger for larger reactant-crowder size ratio, which is in accord with former study. ${ }^{26}$ According to the $\mathrm{MC}$ simulation results, the critical second virial coefficient for entropy-enthalpy 
compensation is different at different packing fractions. This suggests that the association equilibria of the proteins are very sensitive to the crowded environment. Our work can provide general guidelines on how to steer protein interactions in order to cure certain polymerization diseases, and to control the association equilibria of certain types of globular proteins in biochemical engineering.

\section{Acknowledgments}

We thank Professor Jure Dobnikar for helpful discussions. This work was supported by the National Natural Science Foundation of China (Grant Nos. 11232013 and 11472285).

\section{References}

1. H. Zhao, M. T. Magone and P. Schuck, Phys. Biol. 8, 046004 (2011).

2. Z. Liu et al., Biophys. J. 94, 3629 (2008).

3. J. Batra, K. Xu and H. X. Zhou, Proteins 77, 133 (2009).

4. A. P. Minton, Biophys. J. 93, 1321 (2007).

5. A. P. Minton, Biopolymers 99, 239 (2013).

6. J. Wei et al., PLOS ONE 11, e0151159 (2016).

7. H. Reiss, H. L. Frisch and J. L. Lebowitz, J. Chem. Phys. 31, 369 (1959).

8. A. P. Minton and H. Edelhoch, Biopolymers 21, 451 (1982).

9. A. P. Minton, Biophys. Chem. 57, 65 (1995).

10. A. P. Minton, Methods Enzymol. 295, 127 (1998).

11. J. S. Kim and A. Yethiraj, J. Phys. Chem. B 115, 347 (2011).

12. A. Bhattacharya, Y. C. Kim and J. Mittal, Biophys. Rev. 5, 99 (2013).

13. Y. C. Kim, R. B. Best and J. Mittal, J. Chem. Phys. 133, 205101 (2010).

14. Y. C. Kim and J. Mittal, Phys. Rev. Lett. 110, 208102 (2013).

15. Y. C. Kim, A. Bhattacharya and J. Mittal, J. Phys. Chem. B 118, 12621 (2014).

16. M. G. Noro and D. Frenkel, J. Chem. Phys. 113, 2941 (2000).

17. D. L. Pagan and J. D. Gunton, J. Chem. Phys. 122, 184515 (2005).

18. H. J. Liu, S. Garde and S. Kumar, J. Chem. Phys. 123, 174505 (2005).

19. Y. Duda, J. Chem. Phys. 130, 116101 (2009).

20. J. Largo, M. A. Miller and F. Sciortino, J. Chem. Phys. 128, 134513 (2008).

21. D. Costa, P. Ballone and C. Caccamo, J. Chem. Phys. 116, 3327 (2002).

22. E. Zaccarelli et al., Phys. Rev. Lett. 94, 218301 (2005).

23. L. Xu et al., Phys. Rev. Lett. 109, 095702 (2012).

24. F. H. Ree and W. G. Hoover, J. Chem. Phys. 46, 4181 (1967).

25. J. Rosen, Y. C. Kim and J. Mittal, J. Phys. Chem. B 115, 2683 (2011).

26. K. A. Sharp, Proc. Natl. Acad. Sci. USA 112, 7990 (2015). 